ARTICLE

DOI: $10.1038 / \mathrm{s} 41467-017-00946-1$

OPEN

\title{
Lifshitz transition from valence fluctuations in $\mathrm{YbAl}_{3}$
}

Shouvik Chatterjee ${ }^{1,5}$, Jacob P. Ruf ${ }^{1}$, Haofei I. Wei@ ${ }^{1}$, Kenneth D. Finkelstein ${ }^{2}$, Darrell G. Schlom ${ }^{3,4}$ \& Kyle M. Shen ${ }^{1,4}$

In mixed-valent Kondo lattice systems, such as $\mathrm{YbAl}_{3}$, interactions between localized and delocalized electrons can lead to fluctuations between two different valence configurations with changing temperature or pressure. The impact of this change on the momentum-space electronic structure is essential for understanding their emergent properties, but has remained enigmatic. Here, by employing a combination of molecular beam epitaxy and in situ angle-resolved photoemission spectroscopy we show that valence fluctuations can lead to dramatic changes in the Fermi surface topology, even resulting in a Lifshitz transition. As the temperature is lowered, a small electron pocket in $\mathrm{YbAl}_{3}$ becomes completely unoccupied while the low-energy ytterbium ( $\mathrm{Yb}$ ) $4 f$ states become increasingly itinerant, acquiring additional spectral weight, longer lifetimes, and well-defined dispersions. Our work presents a unified picture of how local valence fluctuations connect to momentum-space concepts such as band filling and Fermi surface topology in mixed valence systems.

\footnotetext{
${ }^{1}$ Laboratory of Atomic and Solid State Physics, Department of Physics, Cornell University, Ithaca, NY 14853, USA. ${ }^{2}$ Cornell High Energy Synchrotron Source, Wilson Laboratory, Cornell University, Ithaca, NY 14853, USA. ${ }^{3}$ Department of Materials Science and Engineering, Cornell University, Ithaca, NY 14853, USA ${ }^{4}$ Kavli Institute at Cornell for Nanoscale Science, Ithaca, NY 14853, USA. ${ }^{5}$ Present address: Department of Electrical \& Computer Engineering, University of California, Santa Barbara, CA 93106, USA
} 
K ondo lattice systems host a wide variety of quantum states such as antiferromagnetism ${ }^{1}$, heavy Fermi liquids ${ }^{2}$, hidden order $^{3}$, and unconventional superconductivity ${ }^{4}$, which can often be controlled by modest perturbations using magnetic field or pressure, thereby providing access to quantum phase transitions ${ }^{5-7}$. These states generally emerge from a complex manybody state that is formed by enhanced Kondo coupling between the local rare-earth moments and the band-like conduction electrons at low temperatures. In mixed valence systems ${ }^{8-10}$, this coupling also results in a change of the rare-earth valence, which can be determined by core-level spectroscopies that probe the local chemical environment $(r \text {-space })^{11-13}$, but the implications for the momentum-space ( $k$-space) electronic structure remain poorly understood. To gain insight into the emergent properties of these systems, it is crucial to understand how delocalized carriers and the low-energy momentum-space electronic structure emerge from these local interactions.

Here, we choose $\mathrm{YbAl}_{3}$ as a simple prototypical mixed valence system with two nearly degenerate ytterbium $(\mathrm{Yb})$ valence configurations, $\mathrm{Yb}^{2+}\left(4 f^{14}\right)$ and $\mathrm{Yb}^{3+}\left(4 f^{13}\right)$. The average $\mathrm{Yb}$ valence, $\nu_{\mathrm{f}}$, decreases with temperature, changing by $\sim \Delta \nu_{\mathrm{f}}=-0.05$ from $300 \mathrm{~K}$ to below $T^{*} \approx 34-40 \mathrm{~K}^{11,12,14-16}$, when it becomes a heavy Fermi liquid, attributed to the enhanced Kondo screening at low temperatures ${ }^{17}$. We selected $\mathrm{YbAl}_{3}$ due to its relatively large change in valence as well as its large energy scales, with a reported single ion Kondo temperature $T_{\mathrm{K}} \approx 670 \mathrm{~K}^{17,18}$, which should make these changes observable in momentum space. The lack of a welldefined, pristine surface in cleaved $\mathrm{YbAl}_{3}$ single crystals ${ }^{19}$, however, has previously prevented momentum-resolved measurements of its electronic structure. We have circumvented this problem by synthesizing epitaxial thin films of $\mathrm{YbAl}_{3}$ and its conventional metal analog $\mathrm{LuAl}_{3}$ by molecular beam epitaxy $(\mathrm{MBE})^{20}$ and have combined it with in situ angle-resolved photoemission spectroscopy (ARPES) to directly measure their electronic structure as a function of temperature. Our measurements reveal a strong temperature-dependent change in both the real and momentumspace electronic structure of $\mathrm{YbAl}_{3}$. The local $\mathrm{Yb}$ valence decreases as the temperature is lowered, accompanied by a large shift in the chemical potential which leads to a Lifshitz transition of a small electron pocket at $\Gamma$, along with the emergence of renormalized heavy quasiparticles near the Fermi energy $\left(E_{\mathrm{F}}\right)$. We establish a direct one-to-one correspondence between these observed changes, which we believe to be generic to all mixed valence systems.

\section{Results}

Synthesis and electronic structure. Both $\mathrm{YbAl}_{3}$ and $\mathrm{LuAl}_{3}$ crystallize in a cubic $P m \overline{3} m$ structure where $\mathrm{Yb}$ or $\mathrm{Lu}$ atoms occupy the vertices of the unit cell while $\mathrm{Al}$ atoms occupy the face centers, as illustrated in the inset of Fig. $1 \mathrm{~b}$. $\mathrm{LuAl}_{3}$ has fully occupied $4 f$ orbitals with zero net moment and a lattice constant (4.19 $\AA$ ) closely matched to $\mathrm{YbAl}_{3}(4.20 \AA)$. Thus, $\mathrm{LuAl}_{3}$ serves as an ideal reference compound to understand the light, Al-derived band-like conduction electron states, which are also common to $\mathrm{YbAl}_{3}$. Epitaxial thin films of both $\mathrm{LuAl}_{3}$ and $\mathrm{YbAl}_{3}$ with (001) out-of-plane orientation were synthesized by co-evaporation on $\mathrm{MgO}(001)$ substrates $(4.21 \AA)$ at temperatures of $200-350^{\circ} \mathrm{C}$ and a chamber base pressure below $2 \times 10^{-9}$ Torr. For all films, a 1.2 nm thick aluminum ( $4.05 \AA$ ) buffer layer was deposited at $500{ }^{\circ} \mathrm{C}$, which allowed the growth of continuous, smooth films of $\mathrm{LuAl}_{3}$ / $\mathrm{YbAl}_{3}$ on top. In these studies, we investigated a $30 \mathrm{~nm}$ thick $\mathrm{LuAl}_{3}$ film and a $20 \mathrm{~nm}$ thick $\mathrm{YbAl}_{3}$ film (the $\mathrm{YbAl}_{3}$ was synthesized on top of a $20 \mathrm{~nm}$ thick $\mathrm{LuAl}_{3}$ buffer layer on top of the $\mathrm{Al}$ buffer, which improved the quality of the $\mathrm{YbAl}_{3}$ layers). All films were sufficiently thick so that any photoemission intensity from the buffer layers or substrate and thickness-dependent finite size effects can be ignored. Additional details about the synthesis can be found in "Methods" section as well in ref. ${ }^{20}$.

In Fig. 1, we show Fermi surface maps and the electronic structure from $\mathrm{LuAl}_{3}$ and $\mathrm{YbAl}_{3}$ thin films from the Fermi energy $\left(E_{\mathrm{F}}\right)$ to a binding energy of $10.5 \mathrm{eV}$. For $\mathrm{LuAl}_{3}$, only the Lu $4 f^{13}$ final states are observed, with the $J=7 / 2$ and $5 / 2$ core levels at binding energies of 6.7 and $8.2 \mathrm{eV}$, respectively. Highly dispersive Al-derived bands can be observed in both $\mathrm{LuAl}_{3}$ (Fig. 1c) and $\mathrm{YbAl}_{3}$ (Fig. 1f), which extend from about $6 \mathrm{eV}$ binding energy to near $E_{\mathrm{F}}$. By matching the experimentally determined dispersion of these bands as well the Fermi surface contours measured with both $\mathrm{He} \mathrm{I} \alpha(21.2 \mathrm{eV})$ and $\mathrm{He} \mathrm{II} \alpha$ $(40.8 \mathrm{eV})$ photons to density functional theory (DFT) calculations, we are able to determine our out-of-plane momenta $\left(k_{\mathrm{z}}\right)$ values for both $\mathrm{YbAl}_{3}$ and $\mathrm{LuAl}_{3}$. Due to the lack of strong correlations in $\mathrm{LuAl}_{3}$ (its $4 f$ shell is entirely filled), DFT calculations should accurately describe its electronic structure and indeed we find good agreement between both the DFTcalculated band dispersions as well as Fermi surface contours to the experimentally determined dispersions and Fermi surface from ARPES, assuming an inner potential of $V_{0}-\phi=13.66 \mathrm{eV}$ (Fig. 1a, c and Supplementary Fig. 1). We observe broadly dispersive, primarily Al-derived bands in $\mathrm{YbAl}_{3}$ (Fig. 1f) analogous to those observed in $\mathrm{LuAl}_{3}$, and also found excellent correspondence between the measured electronic structure in $\mathrm{LuAl}_{3}$ and $\mathrm{YbAl}_{3}$ over the entire Brillouin zone (Supplementary Fig. 3) indicating that we are probing a similar $k_{\mathrm{z}}$ as in $\mathrm{LuAl}_{3}$, as one might expect given their highly similar electronic and crystal structures. Using the value of $V_{0}-\phi$, we determine that for $h \nu=21.2 \mathrm{eV}$, we are probing near the zone center, $\Gamma$, $k_{\mathrm{z}}=0 \pm 0.1 \pi / \mathrm{c}$. More details about the $k_{\mathrm{z}}$ determination can be found in Supplementary Note 1.

A two dimensional slice at $k_{\mathrm{z}}=\Gamma$ of the three-dimensional Fermi surface of $\mathrm{LuAl}_{3}$ accesses a multiply connected Fermi surface sheet consisting of electron-like pockets centered at $(0,0)$ and $(\pi, \pi)$, consistent with our ARPES data, shown in Fig. 1a-c. On the other hand, in $\mathrm{YbAl}_{3}$, we clearly observe both the $\mathrm{Yb} 4 f^{13}$ and $4 f^{12}$ final states around $0-2 \mathrm{eV}$ and $6-10.5 \mathrm{eV}$ binding energy, respectively, consistent with its mixed valence character. The near- $E_{\mathrm{F}}$ electronic structure in $\mathrm{YbAl}_{3}$ is, however, significantly modified by a shift in its chemical potential due to the differing average $\mathrm{Lu}$ and $\mathrm{Yb}$ valence and the interaction between the broad, dispersive bands and the renormalized $\mathrm{Yb} 4 f$ states. In the Fermi surface map of $\mathrm{YbAl}_{3}$ (Fig. 1d), large Fermi surface sheets are prominent and centered at zone edges $(\pi, \pi)$.

Evolution of the electron pocket at $\boldsymbol{\Gamma}$. Having discussed the basic electronic structure, we now turn towards its temperature dependence in $\mathrm{YbAl}_{3}$. In Fig. 2a, we show a series of ARPES spectra obtained along $(0,0)$ to $(0, \pi)$ at $k_{\mathrm{z}} \approx \Gamma$ between 255 and $21 \mathrm{~K}$, which establish a clear temperature-dependent shift of the chemical potential $\Delta \mu$ with the $4 f$-derived states moving closer to $E_{\mathrm{F}}$ as the temperature is lowered, consistent with earlier angleintegrated measurements ${ }^{14}$. The most dramatic effect of $\Delta \mu$ is on a small parabolic electron pocket centered at $\Gamma$. At $255 \mathrm{~K}$, the electron pocket can be clearly observed with its band bottom at $40 \pm 5 \mathrm{meV}$ binding energy and a $k_{\mathrm{F}}$ of $0.20 \pm 0.01 \pi / a$. As the temperature is lowered, the electron pocket is lifted in energy and becomes entirely unoccupied around $21 \mathrm{~K}$. Since the pocket is centered at $\Gamma$, its lifting above $E_{\mathrm{F}}$ would then coincide with a Lifshitz transition. To within experimental resolution, the dispersion or effective mass of the electron pocket does not change apart from a rigid shift due to $\Delta \mu$. Furthermore, while the $\mathrm{Yb} 4 f^{13}$ final states also shifted in energy, the $\mathrm{Yb} 4 f^{12}$ states did not shift appreciably with temperature indicating that the $\Delta \mu$ shift arises 
a

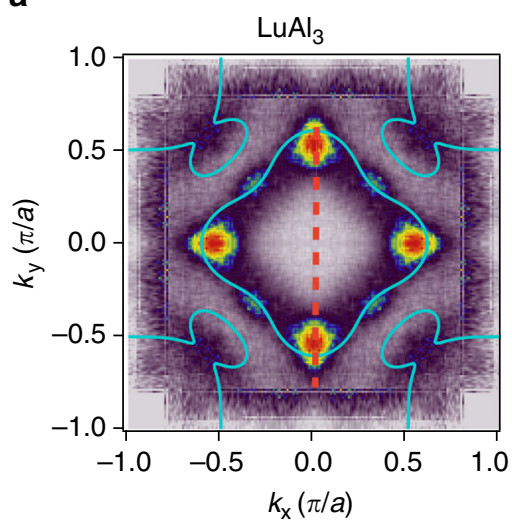

b

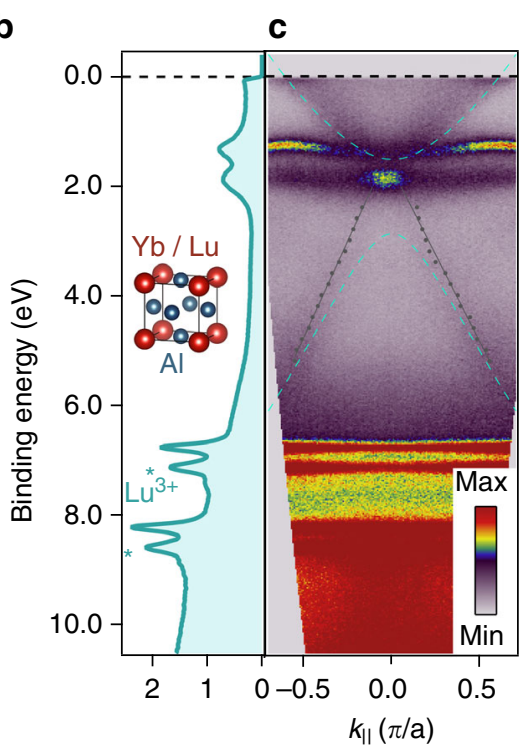

d
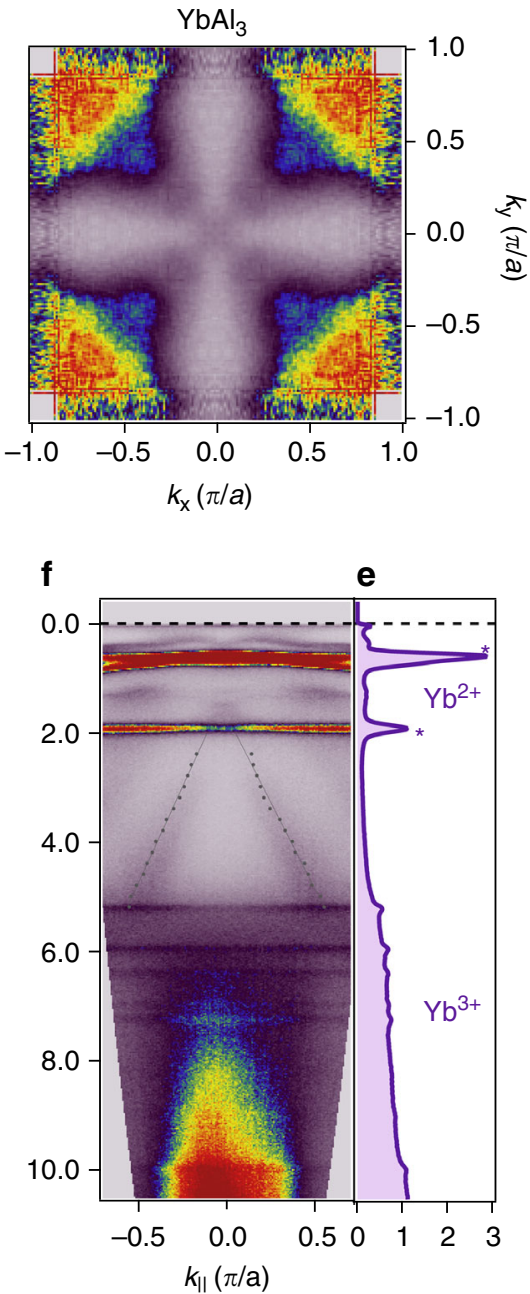

Fig. 1 Electronic structure and Fermi surfaces of $\mathrm{YbAl}_{3}$ and $\mathrm{LuAl}_{3}$. Fermi surface maps and energy distribution curves (EDCs) and $E$ vs. $k$ dispersion for a-c, $\mathrm{LuAl}_{3}$ and $\mathbf{d}-\mathbf{f}, \mathrm{YbAl}_{3}$, all measured with $h \nu=21.2 \mathrm{eV}$ at $21 \mathrm{~K}$. Experimental Fermi surfaces of $\mathbf{a}$, $\mathrm{LuAl}_{3}$ and $\mathbf{d}, \mathrm{YbAl}_{3}$. DFT calculations (green lines) of the Fermi surface topology at $k_{z}=0$ for $\mathrm{LuAl}_{3}$ with $U=0$ are overlaid in $\mathbf{a}$. Momentum-integrated EDCs of $\mathbf{b}$, $\mathrm{LuAl}_{3}$ and $\mathbf{e}$, YbAl 3 , with surface core levels marked as asterisks. $E$ vs. $k$ dispersions for $\mathbf{c}, \mathrm{LuAl}_{3}$ and $\mathbf{f}, \mathrm{YbAl}_{3}$, together with DFT calculations of the band structure in $\mathrm{LuAl}_{3}$ (green) shown in $\mathbf{c}$. The similarity between the dispersion of the light band between 2 and $6 \mathrm{eV}$ in $\mathrm{YbAl}_{3}$ and $\mathrm{LuAl}_{3}$ suggest that both compounds have similar inner potentials, and that both measurements are at $k_{\mathrm{z}}=0 \pm 0.1 \pi / a$

from an alteration in band filling due to the emergence of a Kondo screened many-body state.

We note that the small electron pocket at $\Gamma$ is not reported in previous de Haas-van Alphen ( $\mathrm{dHvA}$ ) studies of $\mathrm{YbAl}_{3}$, which can be explained by the fact that the electron pocket is only occupied at higher temperatures $(T>20 \mathrm{~K})$, whereas dHvA measurements are conducted at low temperatures $(T \approx 20 \mathrm{mK}-1.5 \mathrm{~K})^{18,21}$. Nevertheless, DFT calculations suggest the presence of a quasi-spherical electron pocket at $\Gamma$, but whose size strongly depends on the binding energy of the $\mathrm{Yb} 4 f$ states (Supplementary Fig. 4), and thus the value of $U$ used in the calculations. Furthermore, our observation of the temperaturedependent chemical potential shift may explain the need to artificially shift the chemical potential in previous lowtemperature quantum oscillation experiments of $\mathrm{YbAl}_{3}$ which compared their results to band structure calculations ${ }^{21}$. The large Fermi surface sheets centered at $(\pi, \pi)$ (see Fig. 1d and Supplementary Fig. 2) measured by ARPES would give an oscillation frequency of $\approx 1.0 \pm 0.2 \times 10^{8} \mathrm{Oe}$, which is comparable but somewhat larger than the largest reported quantum oscillation frequency along the (100) direction, $6.51 \times 10^{7}$ Oe.
This discrepancy might be due to the fact that the quantum oscillations are measuring a closed Fermi surface contour at a different $k_{\mathrm{z}}$ than our ARPES measurements at $k_{\mathrm{z}}=0$, which might correspond to an open contour. We also observed another Fermi surface sheet centered at the M point $(\pi, \pi)$ with $40.8 \mathrm{eV}$ photon energy which corresponds to an oscillation frequency of $3.5 \times 10^{7} \pm 1 \times 10^{7}$, roughly consistent with the $\beta$ pocket reported in the dHvA measurements $\left(4.55 \times 10^{7} \mathrm{Oe}\right)$.

In Fig. 2b, we show a series of angle-integrated wide energy valence band in situ $\mathrm{x}$-ray photoemission spectra (XPS) showing a dramatic temperature-dependent change in relative intensity of the $4 f^{13}$ and $4 f^{12}$ final states. As the temperature is lowered, the relative intensity of the $4 f^{13}$ final states increases, while that of the $4 f^{12}$ final states decreases indicating a reduction of the effective $\mathrm{Yb}$ valence in $\mathrm{YbAl}_{3}$ at lower temperatures, which is found to be $\Delta \nu_{\mathrm{f}} \approx 0.05$ from room temperature to below $\approx 45 \mathrm{~K}$, in agreement with previously reported results from bulk samples ${ }^{11,12,14-16}$.

Relation between real-space and momentum-space electronic structure. In Fig. 2c, we make a quantitative comparison between 
a

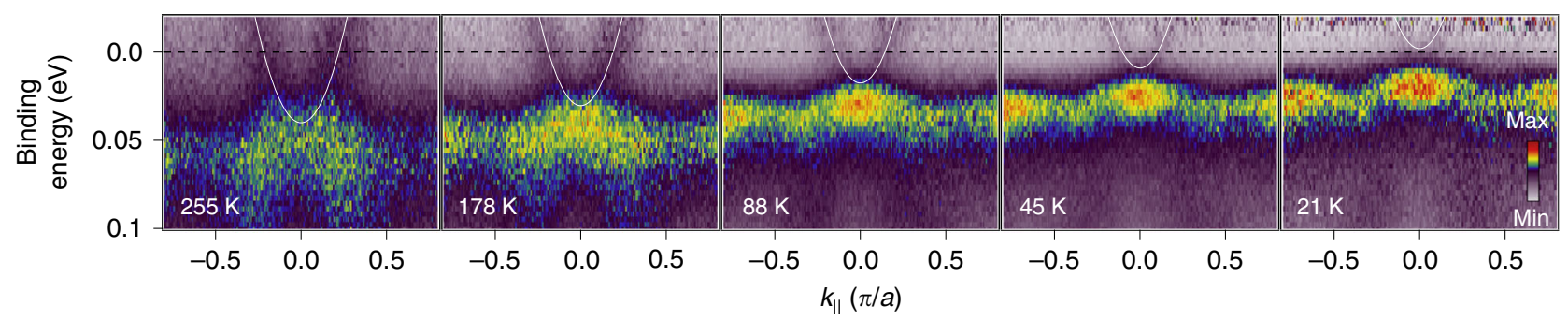

b

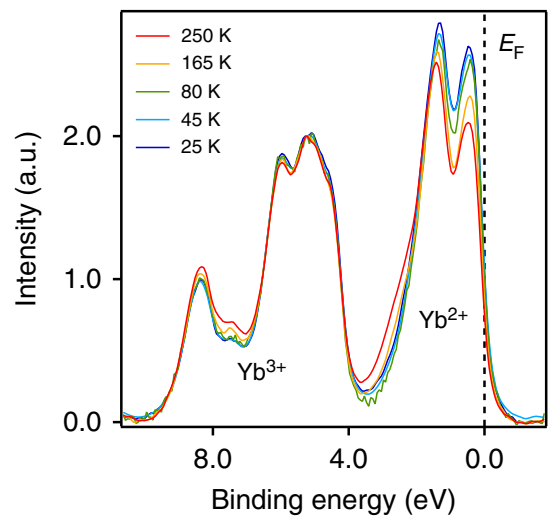

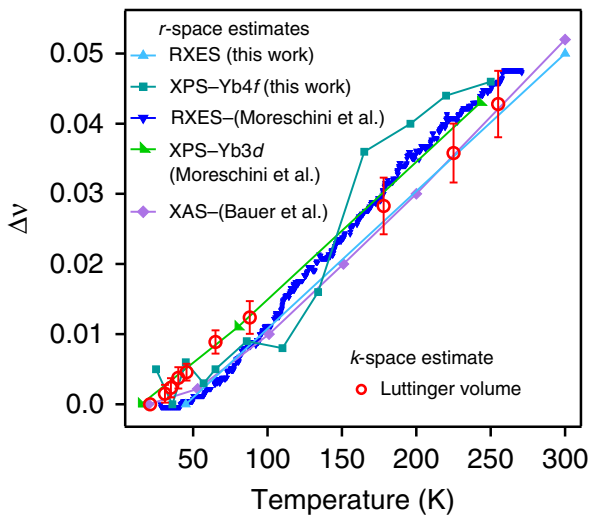

d $r$-space $\quad k$-space

High temperature

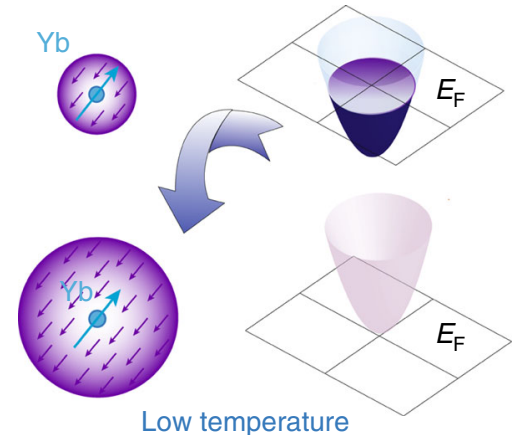

Fig. 2 Correspondence between $r$-space and $k$-space electronic structure in $\mathrm{YbAl}_{3}$. a Evolution of the low-energy electronic structure with temperature. $E$ vs. $k$ dispersions are divided by the corresponding resolution-broadened Fermi-Dirac distribution to emphasize thermally occupied states above $E_{\mathrm{F}}$. White lines are guides to the eye showing evolution of the electron-like pocket centered at $(0,0,0)$. $\mathbf{b}$ XPS spectra showing the temperature-dependent intensity variation of the $4 f^{13}$ and $4 f^{12}$ final states in $\mathrm{YbAl}_{3}$, after Shirley background subtraction ${ }^{37}$ and normalized by the $4 f^{12}$ final state intensity. $\mathbf{c}$ Temperature dependence of the change in Luttinger volume, estimated from the size of the electron pocket at $(0,0,0)$ and of the change in $\mathrm{Yb}$ valence, measured by core-level spectroscopy, revealing a precise one-to-one correspondence. Error bars reflect uncertainty in the Luttinger volume estimation due to a statistical error of one standard deviation in the extracted $k_{\mathrm{F}}$ values from the fits to the Momentum distribution curves (MDCs) taken at $E_{\mathrm{F}}$. $\mathbf{d}$ Schematic illustrating the temperature-dependent relationship between $r$-space and $k$-space electronic structure in $\mathrm{YbAl}_{3}$

the observed change in the temperature-dependent band filling and the estimated change in the $\mathrm{Yb}$ valence from core-level spectroscopy, both in our thin films and previous measurements on $\mathrm{YbAl}_{3}$ single crystals. The change in average $\mathrm{Yb}$ valence in our thin films has been estimated by resonant $\mathrm{x}$-ray emission spectroscopy (RXES) and XPS, details for which can be found in "Methods" section and in Supplementary Note 2. Assuming a spherical geometry $\left(\frac{4}{3} \pi k_{\mathrm{F}}^{3}\right)$ due to its location at $k=(0,0,0)$ and the cubic symmetry, we plot the change in Luttinger volume of the electron pocket $\Delta \nu_{\text {Lutt }}$, vs. the estimated change in $\mathrm{Yb}$ valence $\Delta \nu_{\mathrm{f}}$, from core-level spectroscopy. Without any adjustable parameters or scaling factors, we discover a precise, one-to-one correspondence between $\Delta \nu_{\text {Lutt }}$ from the electron pocket and $\Delta \nu_{\mathrm{f}}$ as a function of temperature. This provides direct microscopic evidence that in $\mathrm{YbAl}_{3}$, the Kondo screening of the $4 f$ moments by the conduction electrons that results in the emergence of composite heavy fermion quasiparticles leads to a Lifshitz transition of the Fermi surface, which is also reflected in the reduction of the average $\mathrm{Yb}$ valence, and should be generic to other mixed valence systems.

A qualitative model of the temperature-dependent changes in both real and momentum-space is presented in Fig. 2d. As the temperature is lowered, the filling of the small electron pocket is gradually reduced as those electrons are transferred into the Kondo screening cloud at the $\mathrm{Yb}$ site leading to the formation of renormalized Kondo screened many-body states ${ }^{22}$ and a reduction of the effective $\mathrm{Yb}$ valence as measured by XPS and RXES studies. This model would explain the direct one-to-one correspondence between the measured changes in both the $\mathrm{Yb}$ valence and the Luttinger volume of the electron pocket as a function of temperature.
We should note that previous studies of other mixed valence systems, such as $\mathrm{YbRh}_{2} \mathrm{Si}_{2}$ have not reported temperaturedependent changes in the band structure or Fermi surface topology ${ }^{23}$, although this may have been because of the much larger $\Delta \nu_{\mathrm{f}}$ in $\mathrm{YbAl}_{3}(0.05$ vs. 0.01$)$ in the accessed temperature range of the experiments, as well as its larger energy scales $\left(T_{\mathrm{K}}=670 \mathrm{~K} \text { vs. } 25 \mathrm{~K}\right)^{23}$.

Evolution of the $\mathrm{Yb} \boldsymbol{4} \boldsymbol{f}$ states. We now discuss the evolution with temperature of the $4 f$-derived heavy bands near $E_{\mathrm{F}}$. In Fig. $3 \mathrm{a}$, we show representative energy distribution curves (EDCs) at different temperatures integrated over the momentum region indicated in Fig. 3d, together with extracted changes of the $4 f$ binding energy, quasiparticle weight, and scattering rate as a function of temperature (Fig. 3b, c). Details of the fitting process along with extended data sets can be found in Supplementary Note 3 and Supplementary Fig. 7. We find a dramatic enhancement of the quasiparticle spectral weight of the $4 f$ bands, consistent with previous measurements by Tjeng et al. ${ }^{11}$, coinciding with a precipitous drop in the scattering rate, which saturates around $T^{*} \approx 37 \mathrm{~K}$, the estimated coherence temperature of $\mathrm{YbAl}_{3}{ }^{20}$ when it becomes a Fermi liquid. The enhancement of the quasiparticle spectral weight and lifetime with decreasing temperature suggests that the screening of the $4 f$ moments by the conduction electrons has nearly saturated around $T^{*}$, and that the Lifshitz transition is coincident with this dramatic change in the $4 f$ spectral function. This is further highlighted by the observation of a $\ln \left(T_{0} / T\right) \geq 255 \mathrm{~K}$ scaling behavior in the integrated spectral 
a

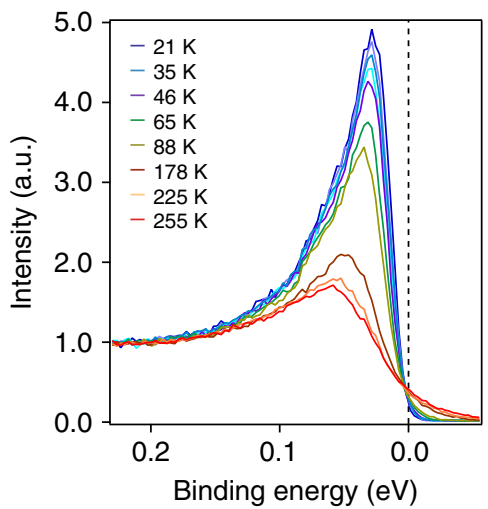

c

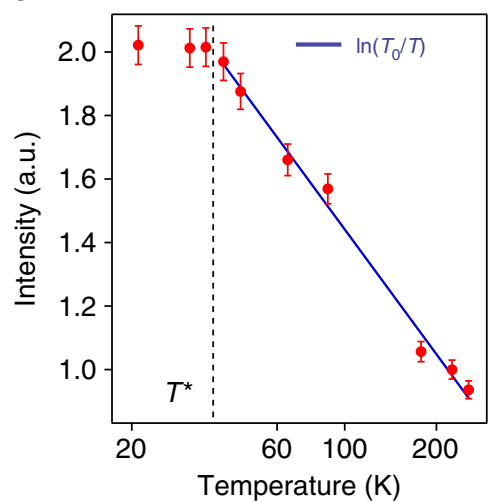

b

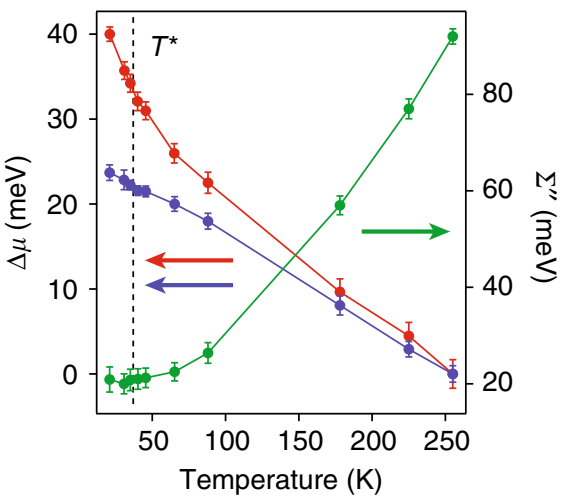

d

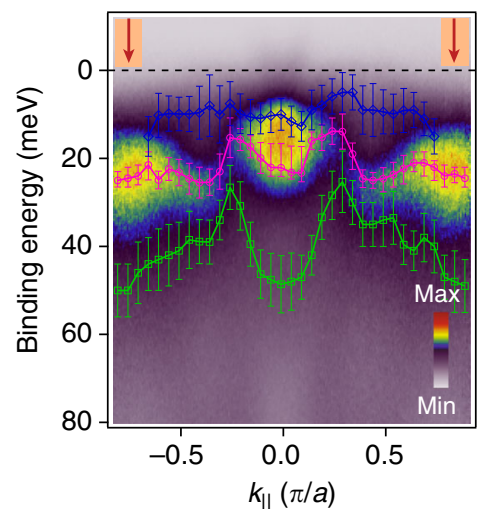

Fig. 3 Evolution of the $\mathrm{Yb} 4 f$ states and crystal field effects in $\mathrm{YbAl}_{3}$. a Evolution of the Kondo resonance peak with temperature, from integrating EDCs over $k$ region highlighted as red in $\mathbf{d}$. $\mathbf{b}$ Change in the chemical potential $(\Delta \mu)$ and in the $4 f$ quasiparticle scattering rate with temperature. $\Delta \mu$ is estimated from the shift in binding energy of the $4 f$-derived heavy band (blue) and the band bottom of the light electron-like pocket at $(0,0)($ red) relative to $255 \mathrm{~K}$. Error bars represent uncertainty due to statistical error of one standard deviation from the fitting process. c Temperature dependence of the integrated spectral weight $(0-0.2 \mathrm{eV})$ of the $4 f$ states which show a $\ln \left(T_{0} / T\right)$ behavior above $T^{*}=37 \mathrm{~K}$. Error bars represent $3 \%$ margin of error. $\mathbf{d}$ High resolution $E$ vs. $k$ plot along $(0,0)-(0, \pi)$ at $21 \mathrm{~K}$ showing dispersive crystal electric field (CEF) split states. Extracted dispersions of the three different CEF split states (shown in blue, pink and green) are superimposed on the image plot. In addition to statistical error of one standard deviation from the fitting process, error bars in $\mathbf{d}$, also include variability in the fit results by holding one and/or two peak positions constant in the multi-peak fitting process

weight, as expected from a two fluid $\operatorname{model}^{22,} 24-26$, until the onset of Fermi liquid behavior at $T^{*}$, when it starts to saturate. The observation of this scaling behavior up to $255 \mathrm{~K}$, the highest temperature accessed in this study, suggests that the hybridization between the local $4 f$ moments and the conduction electrons sets in at a relatively high temperature, even though the Fermi liquid regime exists only below $T^{*}$, consistent with the slow crossover scenario predicted by slave boson mean field calculations ${ }^{27,} 28$. The saturation of the $4 f$ quasiparticle lifetime at $T^{*}$ in our ARPES measurements is also consistent with earlier transport and thermodynamic measurements, which suggested that $T^{*}$ could be related to the formation of coherence in the $4 f$ states $15,17,18$, which we establish spectroscopically. The shift in binding energy of the $4 f$ states is smaller compared to the $\Delta \mu$ measured from the electron-like band, with the discrepancy increasing at lower temperatures, shown in Fig. 3b, indicative of enhanced hybridization between the $4 f$ states and the conduction electrons at lower temperatures that pushes the electron pocket further towards lower binding energy.

Dispersive crystal electric field split states. In addition to the strong temperature dependence of the electronic structure, our measurements clearly show three distinct flat bands close to $E_{\mathrm{F}}$ which acquire significant dispersion at certain $k$ points. Their proximity to $E_{\mathrm{F}}$, the value of their splittings $(\approx 0-30 \mathrm{meV})$, and their narrow bandwidths are all consistent with these being crystal electric field (CEF) split states. The dispersion of the CEF states occur when the light Al-derived bands cross the flat $4 f$ states near $E_{\mathrm{F}}$, lifting the degeneracy of the CEF split branches, as shown in Fig. 3d (also see Supplementary Fig. 8). The observation of three distinct bands is consistent with the bulk cubic symmetry of the $\mathrm{Yb}$ ions, where the $\mathrm{Yb} J=7 / 2$ manifold should split into three crystal field levels $\Gamma_{6}, \Gamma_{7}$, and $\Gamma_{8}{ }^{29}$. While we cannot determine conclusively whether these states are representative of bulk vs. surface $\mathrm{Yb}$ atoms, the values of their splittings $(0-30 \mathrm{meV})$ and bandwidths $(\leq 25 \mathrm{meV})$ from ARPES and the fact that they extend from $E_{\mathrm{F}}$ to a binding energy of $\approx 50 \mathrm{meV}$ are also consistent with reports from bulk-sensitive inelastic neutron scattering which do not observe sharp CEF excitations but rather a broad continuum $(\approx 50 \mathrm{meV})$, since those measurements would average the dispersion of the CEF states over the entire Brillouin zone $\mathrm{e}^{30-32}$.

\section{Discussion}

Our work experimentally provides a unified picture of how local changes of the rare-earth valence impacts the momentum-space 
electronic structure in the prototypical mixed valence system, $\mathrm{YbAl}_{3}$. We have achieved this by combining state-of-the-art materials synthesis and advanced in situ spectroscopy, which should be readily extendable to other Kondo lattice systems or even artificial $f$-electron heterostructures. We have discovered that a Lifshitz transition of a small electron Fermi surface accompanies the change in average $\mathrm{Yb}$ valence, which had hitherto been unanticipated. This discovery underscores how the Kondo screening process can significantly alter $k$-space instabilities of Kondo lattice systems.

\section{Methods}

Film growth and characterizaion. Single crystalline, epitaxial, atomically smooth thin films of (001) $\mathrm{YbAl}_{3}$ and $\mathrm{LuAl}_{3}$ were synthesized on $\mathrm{MgO}$ substrates in a Veeco GEN10 MBE system with a liquid nitrogen cooled cryoshroud at a base pressure better than $2 \times 10^{-9}$ Torr. Prior to growth, $\mathrm{MgO}$ substrates were annealed in vacuum for $20 \mathrm{~min}$ at $800^{\circ} \mathrm{C}$ and a $1-2 \mathrm{~nm}$ thick aluminum (Al) buffer layer was deposited at $500^{\circ} \mathrm{C}$. $\mathrm{Lu} / \mathrm{Yb}$ and $\mathrm{Al}$ were co-evaporated from Langmuir effusion cells at a rate of $\approx 0.4 \mathrm{~nm} / \mathrm{min}$ onto a rotating substrate between 200 and $350^{\circ} \mathrm{C}$ with real-time reflection high-energy electron diffraction (RHEED) monitoring. Due to the co-evaporation growth, the surface termination was not deliberately controlled. After growth, the films were immediately transferred under ultra-high vacuum to an ARPES chamber for measurements. All ARPES data presented in this study were performed on $30 \mathrm{~nm}$ thick $\mathrm{LuAl}_{3}$ thin films with a $1.2 \mathrm{~nm}$ thick $\mathrm{Al}$ buffer layer, or on $20 \mathrm{~nm}$ thick $\mathrm{YbAl}_{3}$ thin films with $20 \mathrm{~nm}$ thick $\mathrm{LuAl}_{3}$ and $1.2 \mathrm{~nm}$ thick Al buffer layers. The ARPES spectra did not show any thickness dependence for $\mathrm{LuAl}_{3} / \mathrm{YbAl}_{3}$ layers that were more than $10 \mathrm{~nm}$ thick, the minimum thickness for this study. For further details regarding film growth and characterization see ref. 20

In situ ARPES and XPS. After growth, thin film samples were immediately transferred within 5 min through ultra-high vacuum into an analysis chamber consisting of a VG Scienta R4000 electron analyzer, VUV5000 helium plasma discharge lamp and a dual anode $\mathrm{x}$-ray source for ARPES and XPS measurements. The base pressure of the analysis chamber was better than $5 \times 10^{-11}$ Torr. ARPES measurements were performed using $\mathrm{He} \mathrm{I} \alpha(h \nu=21.2 \mathrm{eV})$ and $\mathrm{He} \mathrm{I} \alpha(h \nu=40.8$ $\mathrm{eV})$ photons, while $\mathrm{Al} \mathrm{K} \alpha(h \nu=1486.6 \mathrm{eV})$ photons were utilized for collecting XPS data. A polycrystalline gold reference, in electrical contact with the sample was used to determine position of the Fermi level and the energy resolution.

DFT calculations. DFT calculations of the band structure and Fermi surface of $\mathrm{LuAl}_{3} / \mathrm{YbAl}_{3}$ with were performed using full potential linearized augmented plane wave method as implemented in the Wien $2 \mathrm{k}$ software package ${ }^{33}$. The exchange and correlation effects were taken into account within the generalized gradient approximation ${ }^{34}$. Relativistic effects and spin-orbit coupling were included. For $\mathrm{LuAl}_{3}$, we found that an on-site Coulomb repulsion of $U=2.08 \mathrm{eV}^{35}$ would give good agreement between the $\mathrm{Lu} 4 f$ orbitals to the binding energies of the core levels measured in experiment. However, the value of $U$ had no impact on the near- $E_{\mathrm{F}}$ electronic structure in $\mathrm{LuAl}_{3}$. For $\mathrm{YbAl}_{3}$, calculations were performed both with and without application of $U$ to the $\mathrm{Yb} 4 f$ orbitals, which was found to have a significant impact on the near- $E_{\mathrm{F}}$ electronic structure. (Supplementary Fig. 4).

RXES. RXES spectra were collected at the Cornell High Energy Synchrotron Source (CHESS) at the $\mathrm{C} 1$ bend magnet beamline under ring conditions of $5.3 \mathrm{GeV}$ and $100 \mathrm{~mA}$. Incident $\mathrm{x}$-ray radiation was monochromated using a Rh mirror and a sagittal focus double Si(2 20 ) crystal monochromator. The incident energy was calibrated using a $\mathrm{Cu}$ foil. The $\mathrm{x}$-ray emission was monochromated and focused using five spherically bent Ge( 620$)$ crystals in the Rowland geometry by using the CHESS dual array valence emission spectrometer ${ }^{36}$. X-rays were finally collected with a Pilatus $100 \mathrm{~K}$ area detector (Dectris). Use of an area detector offered significant advantages for the current experiment in terms of ease of alignment and reliable background subtraction. Two regions of interest (ROIs) were chosen, one containing more than $95 \%$ of the emission signal and another centered on the first ROI, but four times in size. The larger ROI was used to correct for the average background counts as

$$
I_{\text {corrected }}=I_{\mathrm{ROI}_{1}}-\text { Area }_{\mathrm{ROI}_{1}} \times\left(\frac{I_{\mathrm{ROI}_{2}}-I_{\mathrm{ROI}_{1}}}{\text { Area }_{\mathrm{ROI}_{2}}-\mathrm{Area}_{\mathrm{ROI}_{1}}}\right),
$$

where $\mathrm{ROI}_{1}$ and $\mathrm{ROI}_{2}$ are the larger and smaller ROIs, respectively. $I_{\mathrm{ROI}}$ and Area $_{\mathrm{ROI}}$ denotes the intensity and area corresponding to the region of interest, respectively, while $I_{\text {corrected }}$ is the corrected intensity after background subtraction.

Measured counts were further corrected for variations in incident photon flux by normalizing with the measured incident flux using a $\mathrm{N}_{2}$-filled ionization chamber placed upstream of the sample stage. X-ray emission energy was calibrated measuring the $\mathrm{K}_{\alpha 1}$ and $\mathrm{K}_{\alpha 2}$ lines of a $\mathrm{Cu}$ foil. The overall energy resolution of the setup was determined to be better than $\approx 3 \mathrm{eV}$ measuring quasi- elastic scattering from a polyimide sample. To minimize photodamage, a fast shutter was placed upstream of the ionization chambers that would only open during active data taking, thus minimizing $\mathrm{x}$-ray dosage of the samples. No photodamage was observed even after taking more than four scans (the maximum number of scans used for measurements at a particular spot) at a single spot. The sample was mounted on a closed cycle cryostat with base temperature of $45 \mathrm{~K}$. A helium filled bag covering most of the $\mathrm{x}$-ray path between the sample, analyzer, and detector was placed to reduce air attenuation along the $\mathrm{x}$-ray path.

Data availability. Data that support the findings of this study are available from the corresponding author upon request.

Received: 27 February 2017 Accepted: 8 August 2017

Published online: 11 October 2017

\section{References}

1. Schröder, A. et al. Onset of antiferromagnetism in heavy-fermion metals. Nature 407, 351-355 (2000).

2. Andres, K., Graebner, J. E. \& Ott, H. R. 4f-Virtual-bound-state formation in $\mathrm{CeAl}_{3}$ at low temperatures. Phys. Rev. Lett. 35, 1779-1782 (1975).

3. Mydosh, J. M. \& Oppeneer, P. M. Hidden order, superconductivity, and magnetism: the unsolved case of $\mathrm{Uru}_{2} \mathrm{Si}_{2}$. Rev. Mod. Phys. 83, 1301-1322 (2011).

4. Curro, N. J. et al. Unconventional superconductivity in $\mathrm{PuCoGa}_{5}$. Nature $\mathbf{4 3 4}$ 622-625 (2005)

5. Si, Q., Rabello, S., Ingersent, K. \& Smith, J. L. Locally critical quantum phase transitions in strongly correlated metals. Nature 413, 804-808 (2001).

6. Gegenwart, P., Si, Q. \& Steglich, F. Quantum criticality in heavy-fermion metals. Nat. Phys. 4, 186-197 (2008).

7. Si, Q. \& Steglich, F. Heavy fermions and quantum phase transitions. Science 329, 1161-1166 (2010).

8. Varma, C. M. Mixed-valence compounds. Rev. Mod. Phys. 2, 219-238 (1976).

9. Lawrence, J. M., Riseborough, P. S. \& Parks, R. D. Valence fluctuation phenomena. Rep. Prog. Phys. 44, 1-84 (1980).

10. Parks, R. D. (ed.) Valence Instabilities and Narrow Band Phenomena (Plenum Press, 1977).

11. Tjeng, L. H. et al. Temperature dependence of the Kondo resonance in $\mathrm{YbAl}_{3}$. Phys. Rev. Lett. 71, 1419-1422 (1993).

12. Moreschini, L. et al. Comparison of bulk-sensitive spectroscopic probes of $\mathrm{Yb}$ valence in Kondo systems. Phys. Rev. B 75, 035113 (2007).

13. Kummer, $\mathrm{K}$. et al. Intermediate valence in $\mathrm{Yb}$ compounds probed by $4 f$ photoemission and resonant inelastic x-ray scattering. Phys. Rev. B 84, 245114 (2011).

14. Suga, $\mathrm{S}$. et al. Kondo lattice effects of $\mathrm{YbAl}_{3}$ suggested by temperature dependence of high-accuracy high-energy photoelectron spectroscopy. J. Phys. Soc. Jpn 74, 2880-2884 (2005)

15. Bauer, E. et al. Anderson lattice behavior in $\mathrm{Yb}_{1-x} \mathrm{Lu}_{x} \mathrm{Al}_{3}$. Phys. Rev. B 69, 125102 (2004)

16. Lawrence, J. M., Kwei, G. H., Canfield, P. C., DeWitt, J. G. \& Lawson, A. C. LIII $\mathrm{X}$-ray absorption in $\mathrm{Yb}$ compounds: temperature dependence of the valence. Phys. Rev. B 49, 1627-1631 (1994).

17. Cornelius, A. L. et al. Two energy scales and slow crossover in $\mathrm{YbAl}_{3}$. Phys. Rev Lett. 88, 117201 (2002).

18. Ebihara, T. et al. Dependence of the effective masses in $\mathrm{YbAl}_{3}$ on magnetic field and disorder. Phys. Rev. Lett. 90, 166404 (2003).

19. Wahl, P. et al. Local spectroscopy of the Kondo lattice $\mathrm{YbAl}_{3}$ : seeing beyond the surface with scanning tunneling microscopy and spectroscopy. Phys. Rev. B $\mathbf{8 4}$ 245131 (2011)

20. Chatterjee, S. et al. Epitaxial growth and electronic properties of mixed valence $\mathrm{YbAl}_{3}$ thin films. J. Appl. Phys. 120, 035105 (2016)

21. Ebihara, T. et al. Heavy fermions in $\mathrm{YbAl}_{3}$ studied by the de Haas-van Alphen effect. J. Phys. Soc. Jpn 69, 895-899 (2000).

22. Choi, H. C., Min, B. I., Shim, J. H., Haule, K. \& Kotliar, G. Temperaturedependent Fermi surface evolution in heavy fermion CeIrIn ${ }_{5}$. Phys. Rev. Lett. 108, 016402 (2012).

23. Kummer, K. et al. Temperature-independent Fermi surface in the Kondo lattice $\mathrm{YbRh}_{2} \mathrm{Si}_{2}$. Phys. Rev. X 5, 011028 (2015).

24. Yang, Y.-F. \& Pines, D. Universal behavior in heavy-electron materials. Phys. Rev. Lett. 100, 096404 (2008)

25. Yang, Y.-F., Fisk, Z., Lee, H.-O., Thompson, J. D. \& Pines, D. Scaling the Kondo lattice. Nature 454, 611-613 (2008)

26. Yang, Y.-F. \& Pines, D. Emergent states in heavy electron materials. Proc. Natl Acad. Sci. USA 109, E3060-E3066 (2012).

27. Burdin, S. \& Zlatic, V. Multiple temperature scales of the periodic Anderson model: Slave boson approach. Phys. Rev. B 79, 115139 (2009). 
28. Burdin, S., Georges, A. \& Grempel, D. R. Coherence scale of the Kondo lattice. Phys. Rev. Lett. 85, 1048-1051 (2000).

29. Lea, K. R., Leask, M. J. M. \& Wolf, W. P. The raising of angular momentum degeneracy of $f$-electron terms by cubic crystal fields. J. Phys. Chem. Solids $\mathbf{2 3}$, 1381-1405 (1962).

30. Murani, A. P. Paramagnetic scattering from the valence-fluctuation compound $\mathrm{YbAl}_{3}$. Phys. Rev. B 50, 9882-9893 (1994).

31. Osborn, R., Goremychkin, E. A., Sashin, I. L. \& Murani, A. P. Inelastic neutron scattering study of the spin dynamics of $\mathrm{Yb}_{1-x} \mathrm{Lu}_{x} \mathrm{Al}_{3}$. J. Appl. Phys. 85, 5344-5346 (1999).

32. Christianson, A. D. et al. Localized excitation in the hybridization gap in $\mathrm{YbAl}_{3}$. Phys. Rev. Lett. 96, 0117206 (2006).

33. Blaha, P., Schwartz, K., Madsen, G., Kvasnicka, D. \& Luitz, J. Wien $2 k$, An Augmented Plane Wave Plus Local Orbitals Program for Calculating Crystal Properties (Karlheinz Schwarz, Techn. Universität Wien, Austria, ISBN 39501031-1-2, 2001).

34. Perdew, J. P., Burke, K. \& Ernzerhof, M. Generalized gradient approximation made simple. Phys. Rev. Lett. 77, 3865-3868 (1996).

35. Anisimov, V. I., Solovyev, I. V., Korotin, M. A., Czyzyk, M. T. \& Sawatzky, G. A. Density-functional theory and $\mathrm{NiO}$ photoemission spectra. Phys. Rev. B 48, 16929-16934 (1993).

36. Finkelstein, K. D., Pollock, C. J., Lyndacker, A., Krawcyk, T. \& Conrad, J. Dualarray valence emission spectrometer (DAVES): a new approach for hard X-ray photon-in photon-out spectroscopies. AIP Conf. Proc. 1741, 030009 (2016).

37. Shirley, D. A. High-resolution X-ray photoemission spectrum of the valence bands of gold. Phys. Rev. B 5, 4709-4714 (1972).

\section{Acknowledgements}

We thank Yang Liu, J.W. Allen, J.D. Denlinger, G.A. Sawatzky, and H. Takagi for helpful discussions. This work was supported by the National Science Foundation through DMR-0847385 and the Materials Research Science and Engineering Centers program (DMR-1120296, the Cornell Center for Materials Research), the Research Corporation for Science Advancement (2002S), and by the Gordon and Betty Moore Foundation as part of the EPiQS initiative (GBMF3850). Support from the Air Force Office of Scientific Research was through FA2386-12-1-3013. This work was performed in part at the Cornell NanoScale Facility, a member of the National Nanotechnology Infrastructure Network, which was supported by the National Science Foundation (Grant No. ECCS0335765). This work was also performed in part at the Cornell High Energy Synchrotron Source (CHESS) which is supported by the National Science Foundation and the National Institutes of Health/National Institute of General Medical Sciences under NSF award DMR-1332208. H.I.W. and J.P.R. acknowledge support from the NSF Integrative Graduate Education and Research Traineeship program (DGE-0903653), and H.I.W. also acknowledges support from the NSF Graduate Research Fellowship (DGE-1144153).

\section{Author contributions}

S.C. and K.M.S. conceived the idea. Thin film growth, film characterization, ARPES, XPS, and DFT calculations were performed and analyzed by S.C. RXES was performed by S.C., J.P.R. and H.I.W. with assistance and input from K.D.F, and analyzed by S.C. The manuscript was prepared by S.C. and K.M.S. D.G.S. and K.M.S. supervised the study. All authors discussed results and commented on the manuscript

\section{Additional information}

Supplementary Information accompanies this paper at doi:10.1038/s41467-017-00946-1.

Competing interests: The authors declare no competing financial interests.

Reprints and permission information is available online at http://npg.nature.com/ reprintsandpermissions/

Publisher's note: Springer Nature remains neutral with regard to jurisdictional claims in published maps and institutional affiliations.

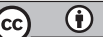

Open Access This article is licensed under a Creative Commons Attribution 4.0 International License, which permits use, sharing, adaptation, distribution and reproduction in any medium or format, as long as you give appropriate credit to the original author(s) and the source, provide a link to the Creative Commons license, and indicate if changes were made. The images or other third party material in this article are included in the article's Creative Commons license, unless indicated otherwise in a credit line to the material. If material is not included in the article's Creative Commons license and your intended use is not permitted by statutory regulation or exceeds the permitted use, you will need to obtain permission directly from the copyright holder. To view a copy of this license, visit http://creativecommons.org/ licenses/by/4.0/.

(C) The Author(s) 2017 